\title{
Clinical Analysis of Pulmonary Nontuberculous Mycobacterial Disease Complicated by Lung Cancer during the Follow-Up Periods
}

\author{
Yoshihiro Kobashi, Makoto Kittaka, Keiji Mouri, Shigeki Kato, Mikio Oka \\ Department of Respiratory Medicine, Kawasaki Medical School, Kurashiki, Japan \\ Email:yoshihiro@med.kawassaki-m.ac.jp
}

How to cite this paper: Kobashi, Y., Kittaka, M., Mouri, K., Kato, S. and Oka, M. (2016) Clinical Analysis of Pulmonary Nontuberculous Mycobacterial Disease Complicated by Lung Cancer during the Follow-Up Periods. Open Journal of Respiratory Diseases, 6, 59-66.

http://dx.doi.org/10.4236/ojrd.2016.64009

Received: October 13, 2016

Accepted: November 27, 2016

Published: November 30, 2016

Copyright $\odot 2016$ by authors and Scientific Research Publishing Inc. This work is licensed under the Creative Commons Attribution International License (CC BY 4.0).

http://creativecommons.org/licenses/by/4.0/

\section{(c) (i) Open Access}

\begin{abstract}
Introduction: The objective of this study was the estimation of the clinical characteristics of patients with pulmonary nontuberculous mycobacterial (NTM) disease complicated by lung cancer during the follow-up periods. Methods: We analyzed the clinical findings of four patients $(2.0 \%)$ complicated by lung cancer during the follow-up periods of over six months at least after the definite diagnosis of pulmonary NTM disease of 202 patients with pulmonary NTM disease experienced in our hospital in the last decade. Results: There were four patients with pulmonary NTM disease complicated by lung cancer and all of them were caused by Mycobacterium avium complex (MAC). They were all elderly male patients and had underlying diseases. Three patients were diagnosed with primary lung cancer and one diagnosed with metastatic lung cancer from colon cancer within 3 years after the diagnosis of pulmonary NTM disease. The treatments for lung cancer were surgical resection for all patients with localized lesions. One patient died due to the worsening of underlying disease and the remaining three survived except for the recurrence of one patient. Conclusion: Although the complication rate of pulmonary NTM disease and lung cancer was a lower percentage $(2.0 \%)$ than in previous reports, the careful follow-up for patients with pulmonary NTM disease without forgetting the possible complication of lung cancer is necessary.
\end{abstract}

\section{Keywords}

Pulmonary Nontuberculous Mycobacterial (NTM) Disease, Lung Cancer, Solitary Nodular Type

\section{Introduction}

Pulmonary nontuberculous mycobacterial (NTM) disease has been recently increasing 
and especially Mycobacterium avium complex (MAC) accounts for $80 \%$ of the identified NTM cases [1]. Among the radiological patterns of pulmonary MAC disease, there is a solitary nodule type for which the prevalence is infrequent. Because the radiological findings are similar, it was thought to be difficult to differentiate the recurrence of pulmonary NTM disease from the complication of lung cancer. However, it has been described that it is possible to differentiate pulmonary NTM disease and lung cancer using bronchoscopy in the recent reports [2] [3]. We investigated the clinical characteristics of patients with pulmonary NTM disease complicated by lung cancer during the follow-up periods after the definite diagnosis of pulmonary NTM disease to obtain the diagnosis of the complications of lung cancer as soon as possible.

\section{Materials and Methods}

The subjects consisted of 4 patients with pulmonary NTM disease complicated by lung cancer $(2.0 \%)$ (primary lung cancer 3, metastatic lung cancer 1) during the follow-up periods. Four patients were extracted from 202 patients with pulmonary NTM disease who both satisfied the diagnostic criteria of ATS [4] and performed the follow-up periods of over six months at least after the definite diagnosis of pulmonary NTM disease in Kawasaki Medical School Hospital (1070 beds) in the last ten decades (2006-2015). On the other hand, we removed the cases which could not perform the follow-up within six months after the definite diagnosis. We retrospectively investigated the backgrounds, laboratory findings, radiological findings, interval from the diagnosis of pulmonary NTM disease to that of lung cancer, treatment, and prognosis for these subjects.

This study was approved by the Ethical Committee of Kawasaki Medical School.

\section{Results}

The main clinical findings of these four patients are shown in Table 1 and Table 2. The age distribution ranged from 72 to 81 years old (average: 77 years old) and all were male. The causative microorganism of pulmonary NTM disease was all MAC ( $M$. intracellulare

Table 1. Clinical findings of pulmonary nontuberculous mycobacterial (NTM) disease complicated by lung cancer.

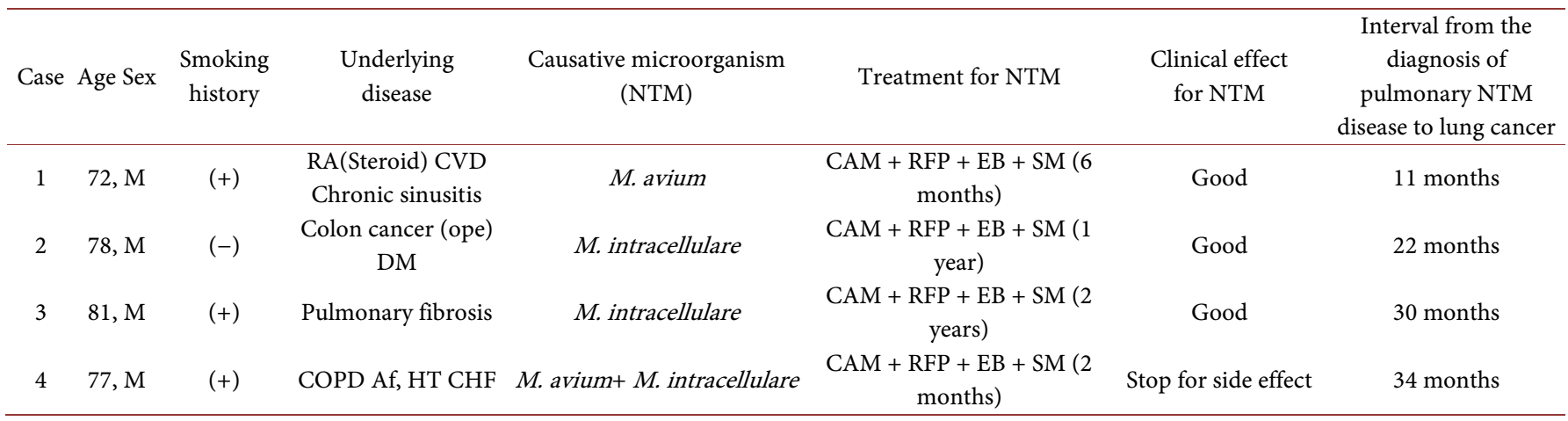

RA: Rheumatoid arthritis, CVD: Cerebral vascular disease, COPD: Chronic obstructive pulmonary disease, DM: Diabetes mellitus, Af: Atrial fibrillation, HT: Hyper tension, CHF: Chronic heart failure, CAM: Clarithromycin, RFP: Rifampicin, EB: Ethambutol, SM: Streptomycin. 
Table2. Clinical findings of pulmonary NTM disease complicated by lung cancer.

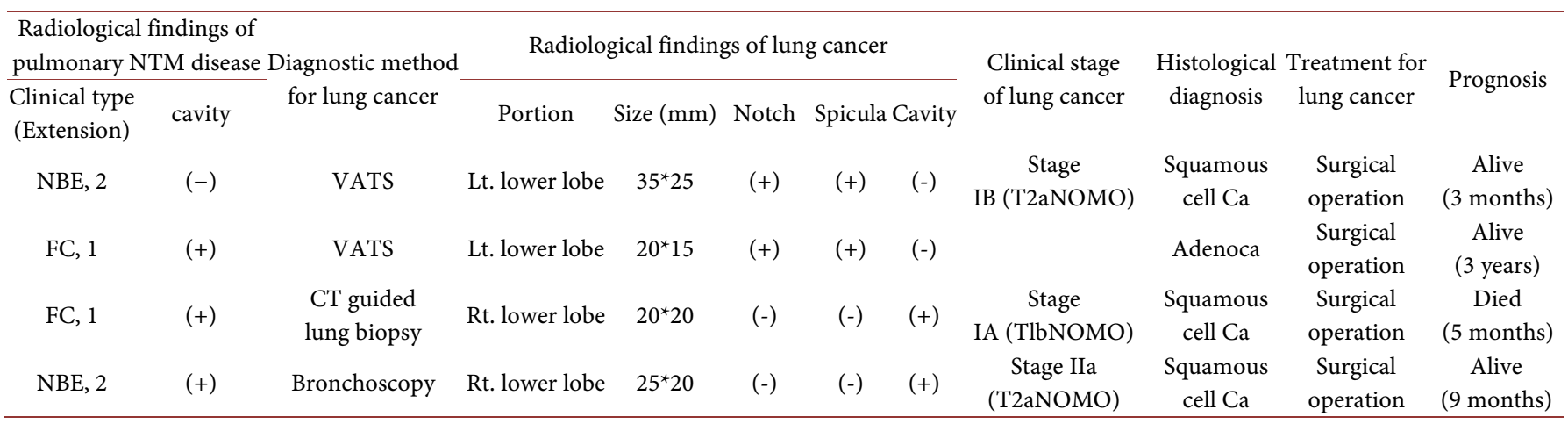

W.N.L.: Within normal limit, FC: Fibrocavitary, NBE: Nodular bronchiectatic, VATS: Video-assisted thoracoscopic surgery, Extension of lesion: 1. Within one-third of unilateral lung field, 2. Within unilateral lung field, 3. Over unilateral lung field.

2, M. avium 1 and $M$. avium $+M$. intracellulare 1$)$. The interval from the diagnosis of pulmonary NTM disease to lung cancer ranged from 11 to 34 months (average: 24.3 months). Regarding the main laboratory findings, the anti-GPL core IgA antibody for MAC was elevated in two of three patients measured and the inflammatory response was mildly positive in one patient. On the other hand, tumor markers were elevated in three of four patients. In the radiological findings of pulmonary NTM disease, the fibrocavitary type was observed in two patients (Figure 1 and Figure 2) and the nodular bronchiectatic type in two (Figure 3 and Figure 4), while cavity lesions were recognized in three patients. The combined chemotherapy for pulmonary NTM disease was initiated for all patients using clarithromycin (CAM), rifampicin (RFP), ethambutol (EB), and streptomycin (SM). Although one patient had to stop the treatment due to side effects (eruption and gastrointestinal symptoms), the remaining three patients showed a good clinical effect.

Concerning the complications of lung cancer, while one patient presented with onenodular lesion in the same lobe as pulmonary NTM disease (Figure 4), the remainingthree patients showed a solitary nodule in the other lobe of pulmonary NTM disease (Figures 1-3). The lung cancer occurred from the peripheral lung field in all patients. The definite diagnosis was obtained from video-assisted thoracoscopic surgery (two patients), transbronchial lung biopsy (one patient), and CT-guided lung biopsy (one patient), respectively. The histological findings of lung cancer were squamous cell carcinoma in three patients with primary lung cancer and adenocarcinoma in one patient with metastatic lung cancer from the colon.

The radiological findings of lung cancer were as follows: the nodule was round, with a clear margin, but notch or spicular formation was recognized in two patients (Figure 1 and Figure 3) and a cavity was observed in two of three patients with primary squamous cell lung cancer (Figure 2 and Figure 4). On PET/CT, because abnormal accumulation was recognized not only in the pulmonary NTM disease lesions but also in those of lung cancer, we could not distinguish the two lesions from the radiological findings. As for lung cancer treatment, all patients were underwent surgical resection.

The prognosis was poor and the condition of one patient worsened due to underlying 


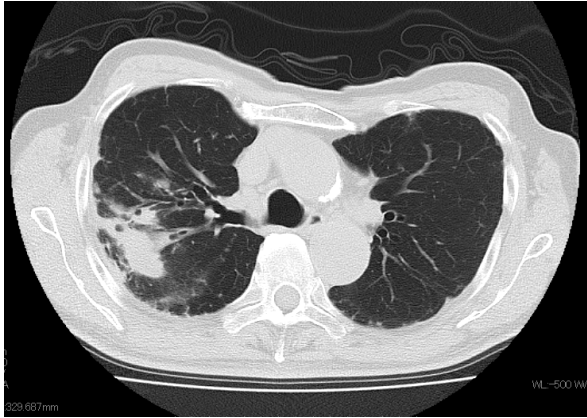

Pulmonary MAC disease

$\mathrm{RFP}+\mathrm{EB}+\mathrm{CAM}+\mathrm{SM}$ (1 year)
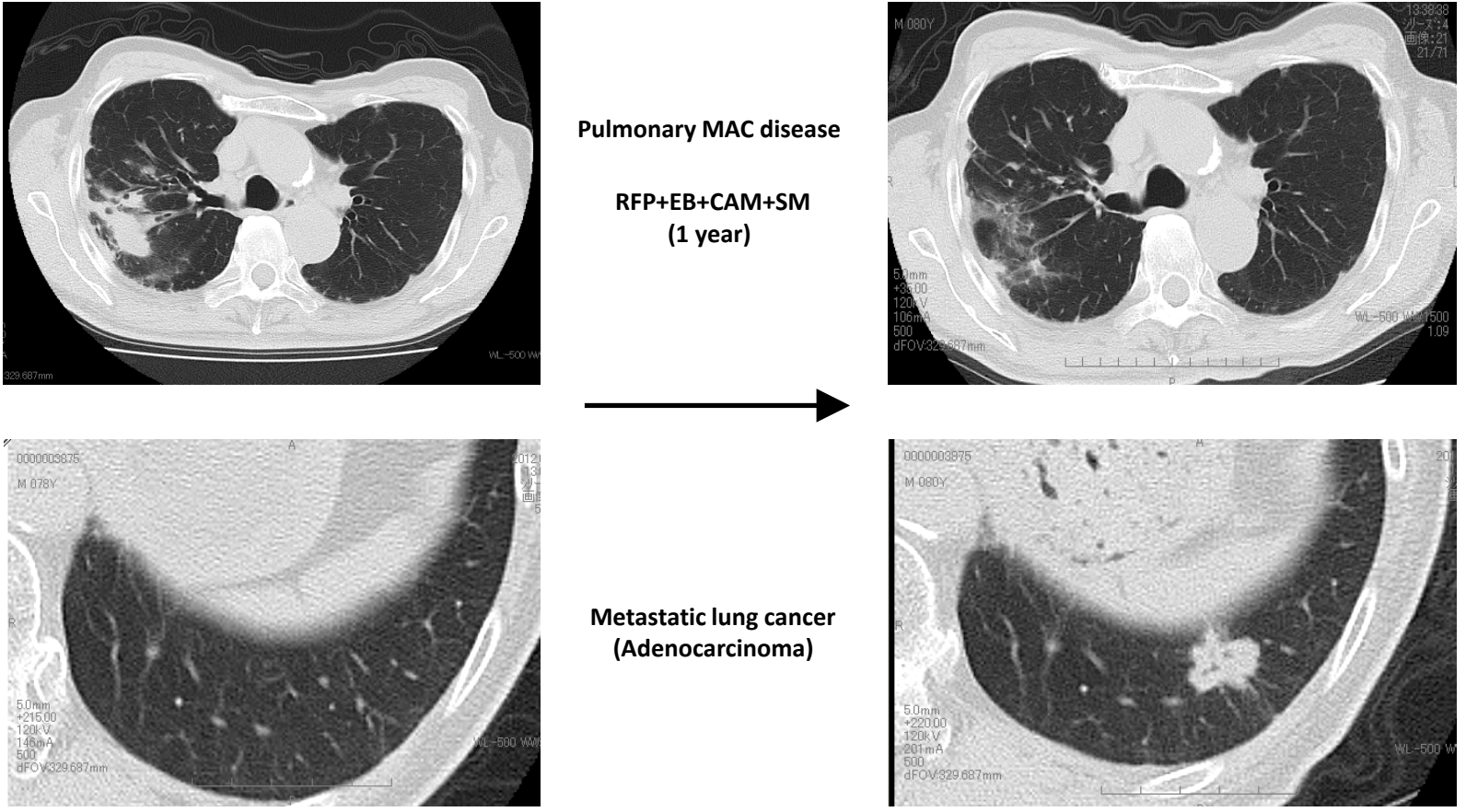

Metastatic lung cancer (Adenocarcinoma)

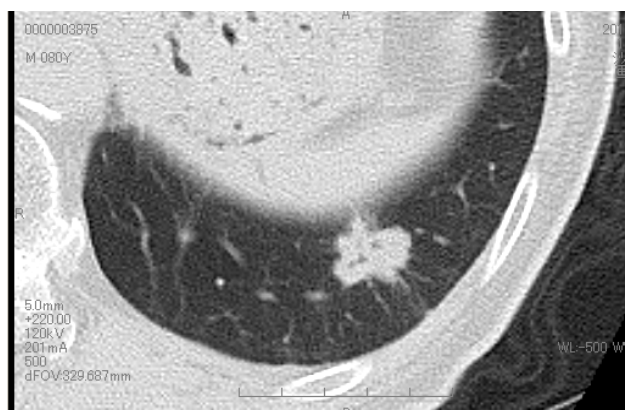

Figure 1. Chest CT at the first visit showed the nodular lesion in the right upper lobe, but there were no lesions in the left lower lobe. Chest CT after the combined chemotherapy including CAM showed the new nodular lesion in the left lower lobe $\left(\mathrm{S}^{10}\right)$.

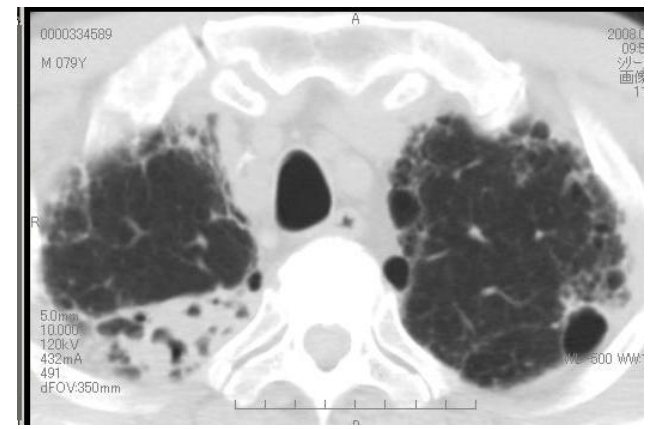

Pulmonary MAC disease

$\mathrm{RFP}+\mathrm{EB}+\mathrm{CAM}+\mathrm{SM}$ ( 2 years)
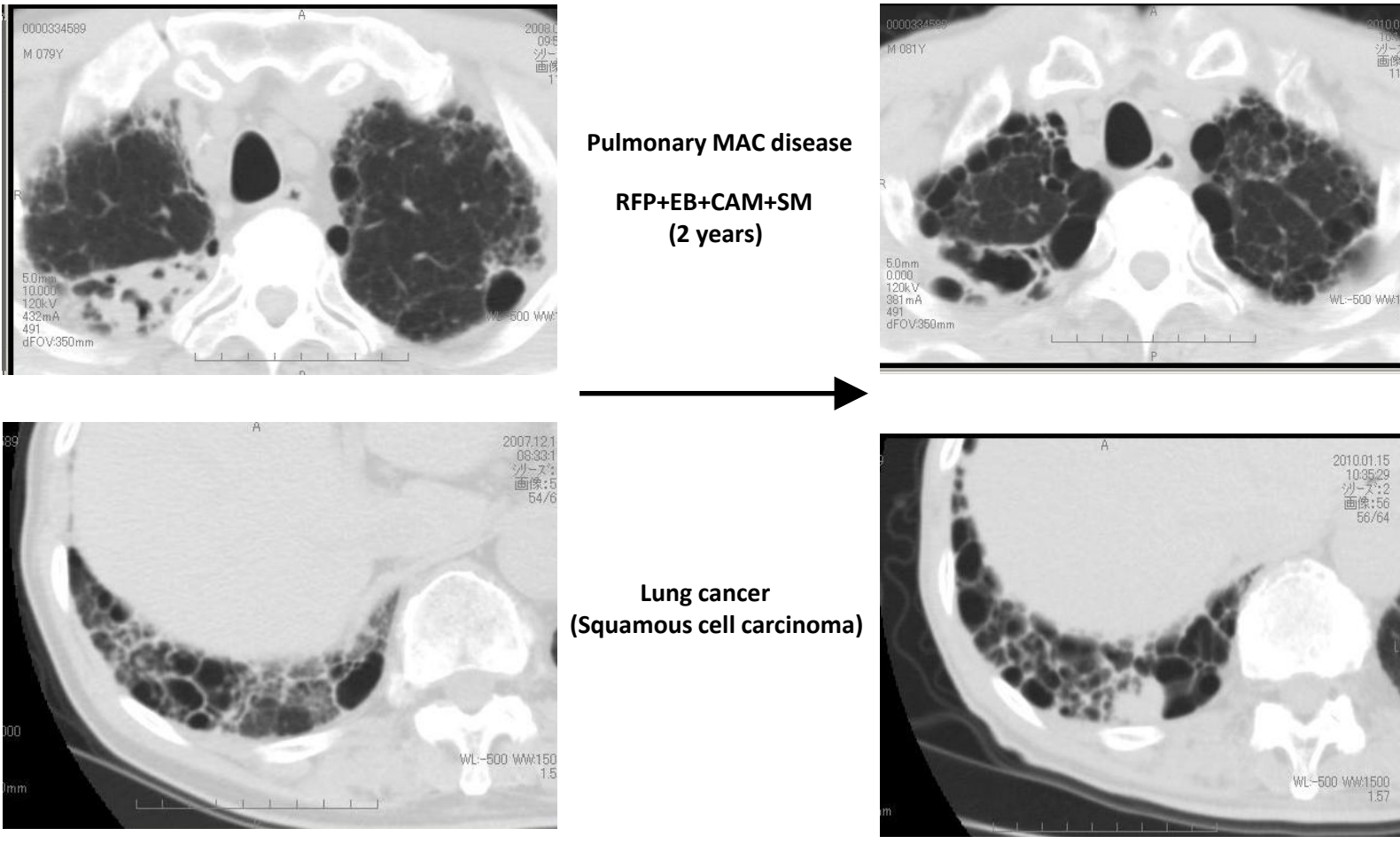

Figure 2. Chest CT at the first visit showed the cavitary lesion in the right upper lobe due to pulmonary MAC disease, but there were no nodular lesions except the reticulonodular shadow due to interstitial pneumonia in the bilateral lower lobes. Chest CT after the combined chemotherapy including CAM showed the improvement of cavitary lesion in the right upper lobe and the appearance of nodular shadow in the right lower lobe. 

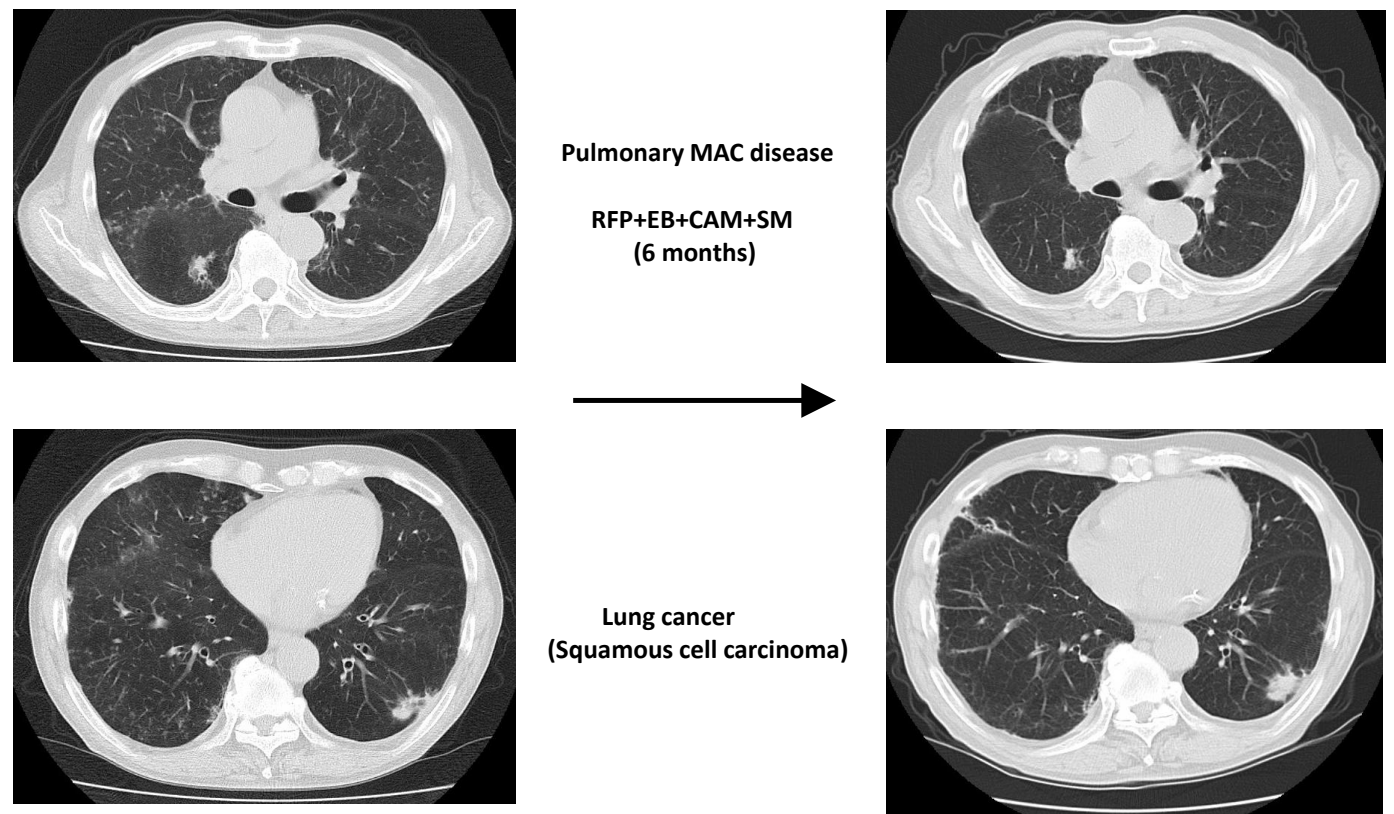

Figure 3. Chest $\mathrm{CT}$ at the first visit showed the small nodular lesions in the right middle lobe and lower lobe with nodular lesion in the right $\mathrm{S}^{6}$ and left $\mathrm{S}^{9}$. Chest CT after the combined chemotherapy including CAM showed the improvement of nodular lesion in the right $S^{6}$ and small nodular lesions in the right lung, but the worsening of left nodular shadow $\left(S^{9}\right)$.
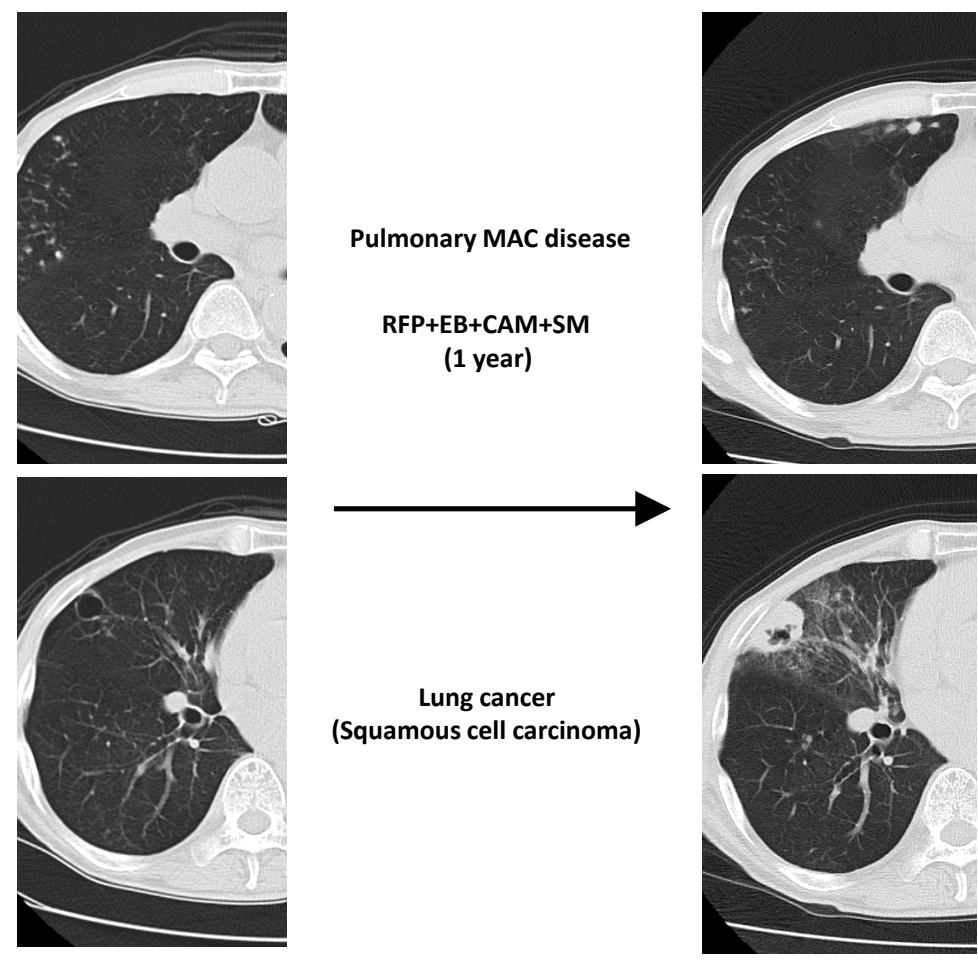

Figure 4. Chest $\mathrm{CT}$ at the first visit showed small nodular lesions with bronchiectasis in the right middle and lower lobes due to pulmonary MAC disease. Chest CT after the stop of the combined chemotherapy due to the side effect showed the appearance of nodular shadows with cavity in the same right middle lobe. 
diseases. Although the other three patients were alive after surgical resection, but one patient showed recurrence within one year.

The clinical findings of the patients with pulmonary NTM disease during the followup periods over six months at least in our hospital are shown in Table 3. Although there was a small number of patients with pulmonary NTM disease complicated by lung cancer, we found the characteristic finding of patients with pulmonary NTM disease complicated by lung cancer after the comparison of the clinical findings of all patients with pulmonary NTM disease to be as follows: 1) all patients were elderly males and had many underlying disease, 2) although the combined chemotherapy including CAM was performed for all patients and the clinical effect to pulmonary NTM disease was comparatively good, the prognosis was poor (the mortality rate was $25 \%$ ) by the influence of underlying disease.

\section{Discussion}

Pulmonary tuberculosis has recently been decreasing, but it has been suggested that pulmonary NTM disease has been increasing. Concerning the study of pulmonary tuberculosis complicated by lung cancer, it was previously described by several reports [5]

Table 3. Clinical findings of the patients with pulmonary NTM disease during the follow-up periods in our hospital.

\begin{tabular}{|c|c|c|}
\hline Clinical finding & & $\mathrm{n}=202$ \\
\hline Age (Mean \pm S.D., years) & & $67.2 \pm 12.5$ \\
\hline Sex (Male: Female) & & 70: 132 \\
\hline Smoking history $(+)$ & & $71(50 \%)$ \\
\hline Underlying disease (+) (with repetition) & & $132(65 \%)$ \\
\hline Respiratory disease & & $48(24 \%)$ \\
\hline Old pulmonary tuberculosis & & 15 \\
\hline COPD & & 12 \\
\hline Non-respiratory disease & & $92(46 \%)$ \\
\hline \multicolumn{3}{|l|}{ Causative microorganisms } \\
\hline M. avium & & $100(50 \%)$ \\
\hline M. intracellulare & & $79(39 \%)$ \\
\hline M. kansasii & & $14(7 \%)$ \\
\hline Others & & $9(4 \%)$ \\
\hline \multicolumn{3}{|l|}{ Radiological findings } \\
\hline \multirow[t]{2}{*}{ Portion } & Bilateral & $141(70 \%)$ \\
\hline & Unilateral & $61(30 \%)$ \\
\hline \multirow[t]{3}{*}{ Extension\# } & 1 & $66(33 \%)$ \\
\hline & 2 & $116(57 \%)$ \\
\hline & 3 & $20(10 \%)$ \\
\hline Cavity & & $127(63 \%)$ \\
\hline \multicolumn{3}{|l|}{ Treatment } \\
\hline Combined chemotherapy & & $131(65 \%)$ \\
\hline Surgical operation & & $9(4 \%)$ \\
\hline \multicolumn{3}{|l|}{ Outcome } \\
\hline Died & & $9(4 \%)$ \\
\hline
\end{tabular}


[6] [7] as follows: 1) most patients were elderly males and had many underlying diseases, 2) most patients had a histological diagnosis of squamous cell carcinoma, 3) the incidence of pulmonary tuberculosis complicated by lung cancer was $1 \%-2 \%$. On the other hand, while the incidence of pulmonary NTM disease complicated by lung cancer was $6 \%-7 \%$ in reports from western countries [8] [9], this was lower (2.5\%) in Japan [7]. In our study, the incidence of pulmonary NTM disease complicated by lung cancer was also low percentage (2.0\%). The clinical characteristics of this study was as follows: i) most of patients were elderly males with a smoking history, ii) the fibrocavitary type was more frequent, iii) lung cancer was located in the neighboring or distant portion of pulmonary NTM disease and it presented as squamous cell carcinoma from the peripheral lung field, iv) it was difficult to distinguish the solitary nodular type of pulmonary NTM disease from lung cancer on radiological findings on chest CT or PET/CT, v) the prognosis was influenced by the performance of surgical resection rather than the clinical effect on pulmonary NTM disease.

In addition, Tamura et al. reported that lung cancer was highly complicated in male patients with pulmonary NTM disease due to M. kansasii, and that both lung cancer and pulmonary NTM disease were located on the same lobe [7]. Lande et al. reported that lung cancer was very complicated for smokers, and that it presented as squamous cell carcinoma in the peripheral lung field [9]. The clinical characteristics of these past reports resembled the results of our study in many findings, but a slight difference was recognized in the background and causative microorganisms. Tamura et al. mentioned that the reason M. kansasii was dominant among the causative microorganisms of pulmonary NTM disease complicated by lung cancer, was because it was commonly found in males with a smoking history and that smoking was an important risk factor for lung cancer [7]. This difference may be due to the number of subjects (202 patients with pulmonary NTM disease performed the follow-up periods over six months at least after the definite diagnosis of pulmonary NTM disease in our hospital) and, especially, pulmonary $M$. kansasii disease accounted for only 19 patients. This small scale study or follow-up period of the patients in this study (over six months) may have also been influenced by the low complication rate (2.0\%) of lung cancer for patients with pulmonary NTM disease compared to other previous reports. As the patients with metastatic lung cancer from the colon and all three patients with pulmonary NTM disease complicated by primary lung cancer were elderly males with a smoking history and with other underlying respiratory diseases, excluding pulmonary NTM disease and primary lung cancer, all cases were squamous cell carcinoma occurring in the peripheral lung field; therefore, it was suspected that smoking history or other underlying respiratory diseases were more strongly related to the appearance of lung cancer.

The clinical disease type was classified into the following five groups: 1) small nodular and bronchiectatic type, 2) fibrocavitary type, 3) solitary nodular type, 4) hypersensitivity type, and 5) disseminated type. Among these groups, most patients with pulmonary NTM (MAC) disease were the small nodular and bronchiectatic type, while the solitary nodular type was rare, but the differentiation of lung cancer is an initial re- 
quirement because all patients with pulmonary NTM disease, excluding one patient with advanced lung cancer, showed a solitary nodular shadow in the peripheral lung field, so it was difficult for us to distinguish a recurrence of pulmonary NTM disease or a complication of lung cancer or pulmonary mycosis. Finally, we reached a definite diagnosis by performing bronchoscopy, positive CT-guided lung biopsy, or VATS for patients without a definite diagnosis.

\section{Conclusion}

Although the incidence of lung cancer complications for patients with pulmonary NTM disease was not so high, even if combined chemotherapy for pulmonary NTM disease was successful, new nodular lesions which were difficult to distinguish from recurrence appeared within a few years. Therefore, careful follow-up for patients with pulmonary NT disease using chest CT is important.

\section{Conflict of Interest}

There are no conflicts of interests regarding this manuscript.

\section{References}

[1] Sakatani, M. (1994) Nontuberculous Mycobacteriosis (NTM) in Japan-Epidemiologic and Clinical Study. Kekkaku, 69, 119-124. (In Japanese)

[2] Daley, C.L. (2012) Mycobacterium avium Complex and Lung Cancer Chicken or Egg Both? Journal of Thoracic Oncology, 7, 1329-1330. https://doi.org/10.1097/JTO.0b013e318265a7ef

[3] Tamura, A., Hebisawa, A., Kusaka, K., et al. (2016) Relationship between Lung Cancer and Mycobacterium avium Complex Isolated Using Bronchoscopy. The Open Respiratory Medicine Journal, 10, 20-28. https://doi.org/10.2174/1874306401610010020

[4] American Thoracic Society (2007) An official ATS/IDSA Statement Diagnosis, Treatment and Prevention of Nontuberculous Mycobacterial Diseases. American Journal of Respiratory and Critical Care, 177, 367-416.

[5] Aoki, K. (1993) Excess Incidence of Lung Cancer among Pulmonary Tuberculosis Patients. Japanese Journal of Clinical Oncology, 23, 205-220.

[6] Kurasawa, T., Takahashi, M., Kuse, F., et al. (1992) Clinical Analysis of Patients with the Complication of Lung Cancer and Active Pulmonary Tuberculosis. Kekkaku, 67, 119-125. (In Japanese)

[7] Tamura, A., Hebisawa, A., Tanaka, T., et al. (2004) Clinical Analysis of Patients with Active Pulmonary Tuberculosis Complicated by Lung Cancer. Kekkaku, 79, 367-373. (In Japanese)

[8] Winthrop, K.L., McNelley, E., Kendall, B., et al. (2010) Pulmonary Nontuberculous Mycobacterial Disease Prevalence and Clinical Features. American Journal of Respiratory and Critical Care, 182, 977-982. https://doi.org/10.1164/rccm.201003-05030C

[9] Lande, L., Peterson, D.D., Gogoi, R., et al. (2012) Association between Pulmonary Mycobacterium avium Complex Infection and Lung Cancer. Journal of Thoracic Oncology, 7, 1345-1351. https://doi.org/10.1097/JTO.0b013e31825abd49 
Submit or recommend next manuscript to SCIRP and we will provide best service for you:

Accepting pre-submission inquiries through Email, Facebook, LinkedIn, Twitter, etc. A wide selection of journals (inclusive of 9 subjects, more than 200 journals)

Providing 24-hour high-quality service

User-friendly online submission system

Fair and swift peer-review system

Efficient typesetting and proofreading procedure

Display of the result of downloads and visits, as well as the number of cited articles

Maximum dissemination of your research work

Submit your manuscript at: http://papersubmission.scirp.org/

Or contact ojrd@scirp.org 